\title{
An anthropometric method for sex determination from the mandible: test on British Medieval skeletal collections
}

\author{
Charlotte Cole, ${ }^{1}$ Constantine Eliopoulos, ${ }^{1}$ Eleni Zorba, ${ }^{2}$ Matteo Borrini ${ }^{1}$ \\ ${ }^{1}$ School of Natural Sciences and Psychology, Liverpool John Moores University, Liverpool, UK; ${ }^{2}$ Department of \\ Forensic Medicine and Toxicology, School of Medicine, National and Kapodistrian University of Athens, Greece
}

\begin{abstract}
Sex determination is a vital part of the analysis of skeletal remains and the creation of biological profiles that aid in identification. The pelvis and skull are the regions usually employed by anthropologists and produce very good results. However, the mandible, being a very durable bone and frequently preserved has not received the attention that other skeletal elements have. There
\end{abstract}

Correspondence: Matteo Borrini, Research Centre in Evolutionary Anthropology and Palaeoecology, School of Natural Sciences and Psychology, Liverpool John Moores University, Byrom Street, Liverpool L3 3AF, UK.

Tel: +44 (0)1512312180.

E-mail: M.Borrini@1jmu.ac.uk

Key words: Skeletal sex determination, mandible, metric sexing.

Acknowledgements: the authors are grateful to the Poulton Project and Gloucester City Museum for making their skeletal collections available for study in the Liverpool John Moores Anthropology Collection. We would also like to thank Professor Joel D. Irish for his useful comments during the preparation of the manuscript.

Contributions: CC: substantial contributions to the acquisition, analysis, and interpretation of data; CE: revising the work critically for important intellectual content; EZ: substantial contributions to the analysis of data; MB: substantial contributions to the conception of the work; final approval of the version to be published.

Conflict of interest: the authors declare no conflict of interest.

Note: no funding has been received for this study. An earlier version of the research has been presented as a poster at the meeting of the British Association of Human Identification in November 2014 and received an award.

Received for publication: 9 November 2016.

Revision received: 18 January 2017.

Accepted for publication: 17 March 2017.

(C) Copyright C. Cole et al., 2017

Licensee PAGEPress, Italy

Journal of Biological Research 2017; 90:6380

doi:10.4081/jbr.2017.6380

This article is distributed under the terms of the Creative Commons Attribution Noncommercial License (by-nc 4.0) which permits any noncommercial use, distribution, and reproduction in any medium, provided the original author(s) and source are credited. are some morphological methods for sexing the mandible, however metrics are considered to be more objective and easier to replicate. This study uses the measurements of the bimental breadth and the corpus thickness of the mandible. Univariate and multivariate analysis was carried out to create discriminant function equations. These equations can be used to sex a mandible with overall accuracy rates as high as $77.3 \%$. The results of the present research are similar to those of other studies and indicate that mandibular metrics can be relied upon for sex determination, especially in cases where other elements are not preserved.

\section{Introduction}

Sexual dimorphism is exhibited in human adult skeletons in both size and shape. Determining the sex of skeletal remains is important as several aspects of an anthropological analysis such as age and stature, rely on the sex of an individual being known. ${ }^{1}$ Dimorphism is used to determine the sex of an individual for the purpose of creating biological profiles for unidentified skeletal remains in forensic cases, but also in palaeodemographical contexts in order to draw conclusions about past populations. A variety of metric and non-metric methods have been developed to determine sex in human skeletal remains. ${ }^{2}$ The objective of these studies was to develop methods that in addition to being accurate, they are easy to use, and are not subject to inter- and intra-observer error.

The pelvis provides the highest rate of accuracy for sex determination in adults that can reach $96 \% .^{3,4}$ An example of such a method is that developed by Phenice, ${ }^{5}$ which is widely used and has been tested on various populations. The skull, after the pelvis, is the skeletal region most frequently used for sex determination. However, Weiss ${ }^{6}$ states that there is a bias that leads to females being misidentified as males due to past populations exhibiting larger, more robust crania. He goes on to warn researchers who use the skull as a sole means for sex determination to do so with extreme caution. Walker ${ }^{7}$ devised a method that uses morphological traits and created a five-point ordinal graded system of morphological observations on the cranium and mandible. As an alternative to morphological methods, discriminant function analysis is also used, by employing cranial measurements to determine sex and is considered to be more objective. In addition, it has been demonstrated that metric methods have lower intra- and interobserver error rates. One of the disadvantages however, is that discriminant function analysis is highly population-specific and can cause high error rates when used for cross-population observations. ${ }^{3,8}$ Another limitation with using the cranium for sexing is that it is often fragmented, both in archaeological and modern contexts. In contrast, the mandible is generally more durable and thus 
is usually better preserved. ${ }^{9,10}$ One of the morphological sexing methods of the mandible that can be used in isolation is the posterior mandibular ramus flexure. ${ }^{11} \mathrm{~A}$ basic requirement of this method is that the posterior part of the mandible is preserved, which is not always the case as the anterior part is more robust and has a better preservation rate. ${ }^{12}$ The accuracy rates of the original study were claimed to have been as high as $99 \%$, surpassing both the skull and pelvis in correct sex determination. However, subsequent studies have failed to achieve such high accuracy rates. ${ }^{13,14}$

In addition, archaeological contexts demonstrate that the pelvis and cranium may be fragmented or may not be present as a result of funerary customs, differential preservation, improper recovery and curation as well as numerous other taphonomic factors. It is therefore essential to be able to obtain information from a variety of skeletal elements by taking a combination of several measurements from one bone, or a series of measurements from different bones. ${ }^{15,11}$ The research into the determination of sex from skeletal elements has proven successful in many bones including: the hyoid, ${ }^{16}$ ulna, ${ }^{17}$ sternal end of the rib, ${ }^{18}$ metacarpals, ${ }^{19}$ and the metatarsals. ${ }^{20}$.In regards to sex determination from the mandible, two recent studies have been conducted by Franklin et al. ${ }^{21}$ and Kumar and Lokanadham, ${ }^{22}$ with accuracy rates ranging from $75 \%$ to $84 \%$. Both of these studies employ several measurements which require that the mandible is intact.

The purpose of this study is to explore the feasibility of developing a new method using only two measurements of the mandible to make determination of sex more objective, accurate and reliable. This is particularly important for cases of extensive bone fragmentation and cremations, where traditional sex indicators may not be available. This project can be considered to be a pilot study on this topic.

\section{Materials and Methods}

The osteological material selected for this study originates from two British Medieval sites, Poulton and Gloucester. ${ }^{23,24}$ Both collections are currently housed at Liverpool John Moores University (LJMU). The final selection of suitable specimens included 76 skeletons across both sites: 16 males and 24 females were selected from Poulton, 24 males and 12 females from Gloucester. Inclusion criteria were limited to the mandibles belonging to adults and absence of any pathologies that would have affected the measurements. Any postmortem alteration to the mandibles was not taken into consideration, unless it interfered with the accuracy of the measurements. The specimens from both collections were grouped together, classified as British Medieval. Sex was not known, but was estimated using a multifactorial analysis by experienced LJMU staff. ${ }^{25}$ All specimens possessed both mental foramina; only two cases showed multiple foramina on one side of the mandible and the true foramen was anatomically determined.

\section{Measurements}

For the purpose of this study, two measurements were taken from the mandible: bimental breadth and corpus thickness of the left and right sides (Figure 1). Bimental breadth is the linear distance between the medial borders of the mental foramina. ${ }^{26}$ The bimental breadth was measured for all 76 skeletal samples using digital sliding callipers ( $c a .0 .01 \mathrm{~mm}$ ). The corpus thickness is the maximum measurement in the region of the mental foramen perpendicular to the long axis of the mandibular body according to
Martin and Saller ${ }^{26}$ and Buikstra and Ubelaker ${ }^{25}$ The corpus thickness was also measured using digital callipers. The left corpus thickness was measured in 73 specimens and the right corpus in 70 specimens due to post-mortem damage. All measurements were taken in millimetres $(\mathrm{mm})$. In order to ensure the consistency of the measurements and minimise inter-observer error, an osteometric protocol was adopted. ${ }^{27}$

\section{Intra-observer error}

A total of 25 (approximately $30 \%$ ) of skeletons were randomly selected for the purpose of determining the intra-observer error for the measurements. The bimental breadth, left corpus thickness and right corpus thickness were recorded twice on separate days using digital callipers and employing the same methodology. The second set of measurements was taken two weeks after the first, by the same observer and under the same conditions.

\section{Statistical tests}

IBM SPSS statistics (IBM Inc. version 21 for Windows) software was used to carry out the statistical analysis. Normality tests showed that the data follow a normal distribution. Descriptive statistics, Student's t-tests and discriminant function analysis were performed. Intra-observer error was determined using a paired ttest for significant difference in the mean bimental breadth and corpus thickness between the initial and repeated measurements. The left and right corpus thickness measurements were tested using a paired $t$-test to ascertain whether there is a significant difference between the two sides. A lack of differences between left and right sides would allow the use of an average corpus thickness value. To determine whether the two measurements are sexually dimorphic, an independent $t$-test was performed to detect possible differences between males and females in the medieval sample used in this study.

Discriminant function analysis was performed to determine the groupings between males and females within a British Medieval skeletal population. Three discriminant functions were performed, both univariate and multivariate. The discriminant functions performed were: Function 1: Bimental Breadth; Function 2: Average Corpus Thickness; Function 3: Bimental Breadth and Average Corpus Thickness.

The discriminant function analysis could not be performed on

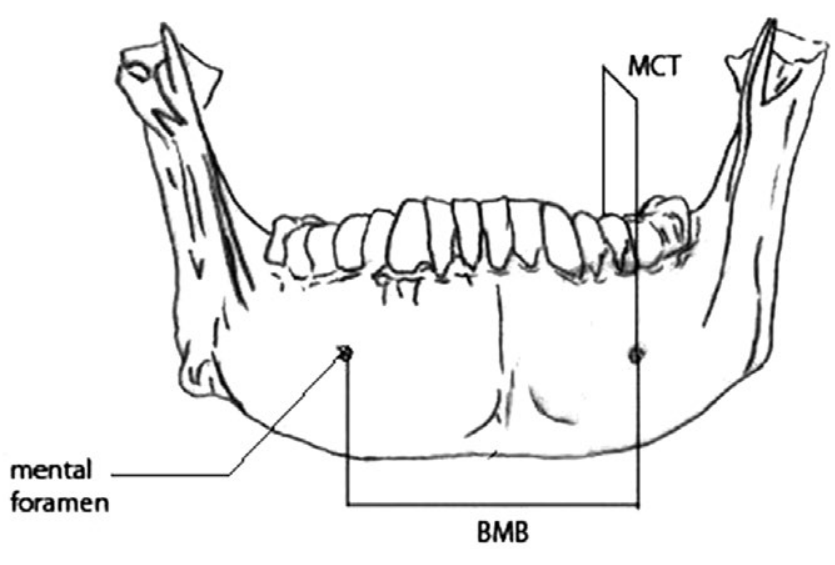

Figure 1. Measurements for bimental breadth (BMB) and mandibular corpus thickness (MCT) (drawing by F.G. Borrini). 
all individuals because of missing values due to damage in the area near the mandibular foramen. The analysis was only carried out for the individuals without missing data that is required for specific functions.

The discriminant function used was:

$\mathrm{F}(\mathrm{X})=\mathrm{C}+\mathrm{U}_{1} \mathrm{X}_{1}+\mathrm{U}_{2} \mathrm{X}_{2}+\ldots . .+\mathrm{U}_{0} \mathrm{X}_{0}$

$\mathrm{F}(\mathrm{X})=$ Discriminant function score

$\mathrm{C}=$ Constant

$\mathrm{U}=$ Unstandardized coefficients of $\mathrm{X}$

$\mathrm{X}=$ Variables

The canonical discriminant functions were then used to create the equation by which to determine sex using the mandibular metrics tested in this study. The sectioning point was found using the group centroids:

Sectioning Point $=$ Male group centroid + Female group centroid $/ 2$

\section{Results}

One of the first tests to be performed was a t-test in order to determine whether there are any side differences for the corpus thickness measurement. The results indicated that no statistically significant differences exist $(t(67)=-0.280, \mathrm{P}=0.780)$. Therefore, all further analyses use the average corpus thickness measurements for each skeleton.

\section{Intra-observer error}

As the measurements were normally distributed it was decided to use the $t$-test. This is a robust test that can be used even when not all of the assumptions are met, as long as the samples are of similar size. The paired $t$-test for significance shows that the bimental breadths did not show a statistically significant difference between the first and second set of measurements taken. Similarly, the corpus thickness test shows that there was no significant difference in the two sets of measurements.

\section{Sexual dimorphism}

Independent $t$-tests were used to determine whether there is a statistically significant difference between males and females for both the bimental breadth and the average corpus thickness measurements. An independent t-test shows that females have a mean bimental breadth $(42.75 \pm 2.09 \mathrm{~mm})$ that is statistically significantly smaller than the mean bimental breadth of the males $(45.75 \pm 2.74 \mathrm{~mm})$, $t(74)=-5.312, \mathrm{P}<0.001$ (Table 1). Likewise, it is shown that females have a mean corpus thickness $(10.52 \pm 1.01 \mathrm{~mm})$ that is statistically significantly smaller than the mean corpus thickness of the males $(11.54 \pm 1.52 \mathrm{~mm}), t(66.384)=-3.443, \mathrm{P}=0.001$ (Table 1).

\section{Discriminant function analysis}

The discriminant function analysis provided unstandardized coefficients and group centroids (Table 2). Sex determination requires a formula in order to acquire a discriminant function score. To generate this score the variables are multiplied with their respective unstandardized coefficients and the results are added along with the constant. The discriminant function score is then compared to the sectioning point in order to determine the sex of the skeletal specimen. If the score is below the sectioning point the individual is female, if the score is above the sectioning point then the individual is male.

Using the information in Table 2 the discriminant equations for functions 1-3 were generated:

$$
\begin{aligned}
& \mathrm{F}(1)=[0.408 * \text { Bimental Breadth }(\mathrm{mm})]-18.072 \\
& \text { Female }<-0.032<\text { Male }
\end{aligned}
$$

Table 1. Descriptive statistics for mandibular measurements and significance of differences in the values of the British Medieval sample used in this study (all values in $\mathbf{m m}$ ).

\begin{tabular}{lccccc} 
Measurement & \multicolumn{2}{c}{ Female } & \multicolumn{2}{c}{ Male } & P-value \\
& Mean & SD & & SD & $<0.001$ \\
Bimental breadth & 42.75 & 2.09 & 45.75 & 2.74 & 0.001 \\
\hline Average corpus thickness & 10.52 & 1.00 & 11.54 & 1.52 & \\
\hline
\end{tabular}

Statistically significant at $\mathrm{P}<0.05$.

Table 2. Canonical discriminant function coefficients for mandibular measurements.

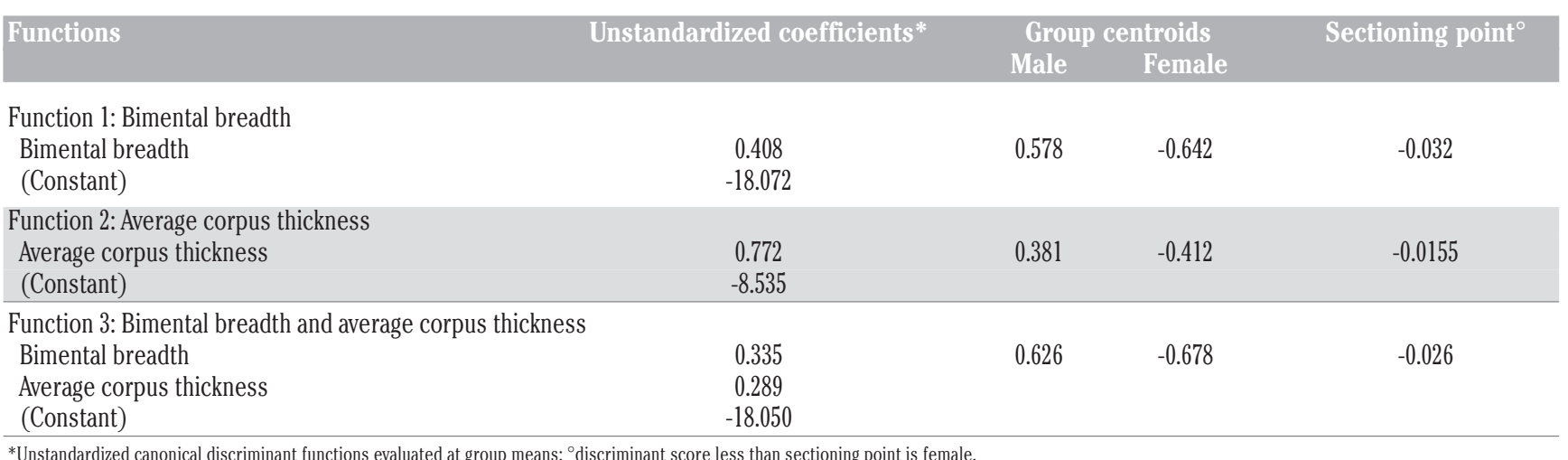


$\mathrm{F}(2)=[0.772 *$ Average Corpus Thickness $(\mathrm{mm})]-8.535$

Female $<-0.0155<$ Male

$\mathrm{F}(3)=[0.335 *$ Bimental Breadth $(\mathrm{mm})]+[0.289 *$ Average Corpus thickness (mm)] -18.050

Female $<-0.026<$ Male

A univariate (functions 1 and 2) and multivariate (function 3) approach was taken to determine the accuracy rate of using the bimental breadth and the corpus thickness in determining the sex of human skeletal remains. The discriminant function analysis uses the measurements to determine and predict the groupings of males and females within the sample. These predictions are used to identify the percentage of correctly classified specimens and the accuracy rates of the discriminant function (Table 3).

The average corpus thickness was plotted against bimental breadth to observe the distribution between males and females. Figure 2 shows the sexes according to the anthropological assessment carried out by morphological and metric methods of the pelvis and skull, while Figure 3 shows the sexes determined by the discriminant function analysis (function 3).

\section{Discussion}

Skeletal sex determination is usually a straight-forward assessment for an experienced anthropologist. However, there are cases where the state of preservation of the remains does not allow for this part of the analysis to be completed. It is therefore essential that in addition to the traditional morphological methods using the pelvis and skull, more skeletal elements are used. This is particularly important in cases of advanced fragmentation or cremation from both ancient/historical and modern contexts. While fragmentation is common in archaeological settings, cremation can be found in both ancient and modern funerary practices. It is also not uncommon for murderers to try to conceal their crimes by using fire in order to destroy evidence, including the bodies of victims. ${ }^{28}$ The potential of using measurements of the mandible has been explored in the present study. Several authors have suggested that mandibular metrics may be a viable solution for the determination of sex. ${ }^{29,21,22}$ Survival of the anterior portions of the mandible is common in most cases, as opposed to the posterior parts which are usually affected early on in the process of cremation. ${ }^{30}$

The results of this study are promising, with the discriminant function analysis showing that both bimental breadth and corpus thickness measurements can be used to determine the sex of an individual successfully and accurately. The univariate analysis showed that function 1 using only the bimental breadth $(73.7 \%)$ is more accurate than function 2 using only the corpus thickness

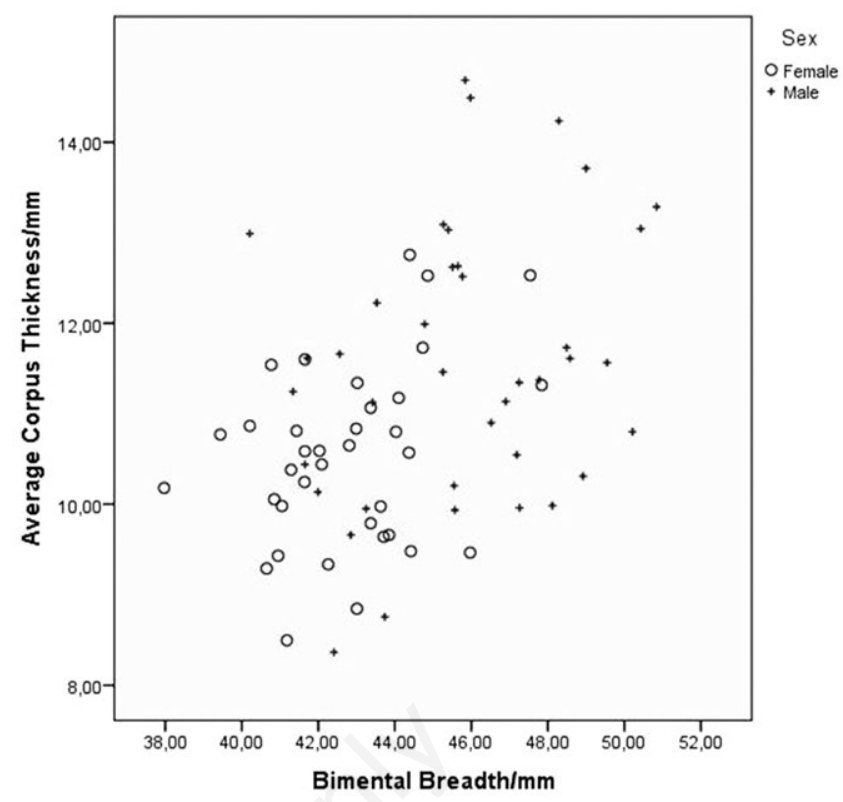

Figure 2. The distribution of sexes according to the anthropological assessment carried out by morphological and metric methods of the pelvis and skull.

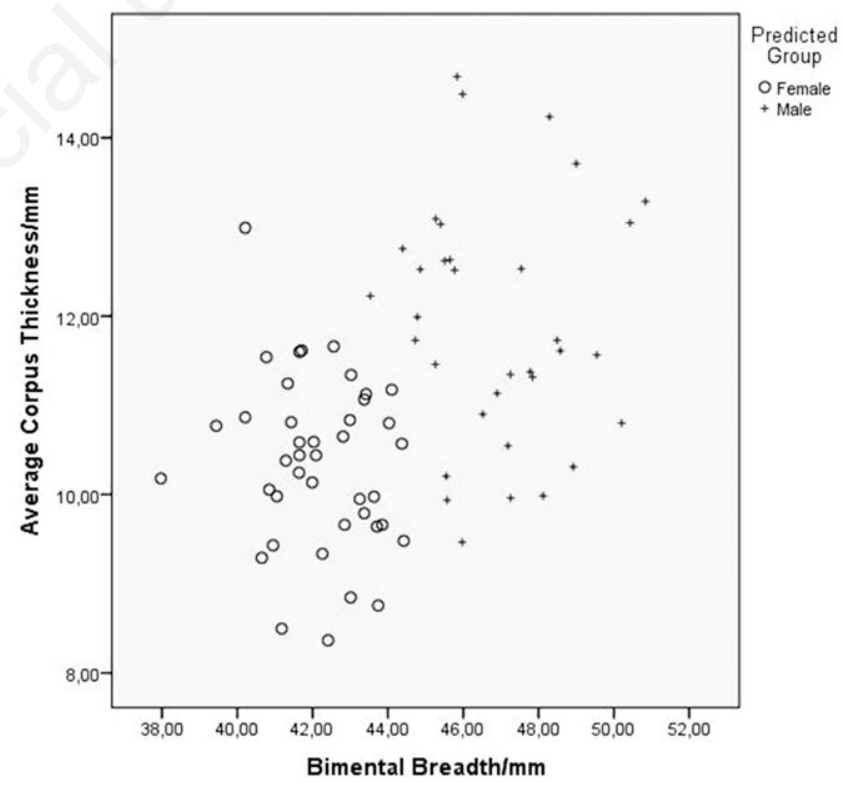

Figure 3. The distribution of males and females based on predicted group membership from discriminant function analysis.

Table 3. Accuracy of classification results of the sample.

\begin{tabular}{|c|c|c|c|c|c|}
\hline \multirow[t]{3}{*}{ Functions } & \multicolumn{4}{|c|}{ Predicted group membership } & \multirow[t]{3}{*}{ Total average (\%) } \\
\hline & \multicolumn{2}{|c|}{ Male } & \multicolumn{2}{|c|}{ Female } & \\
\hline & $\mathbf{N}$ & $\%$ & $\mathbf{N}$ & $\%$ & \\
\hline Bimental breadth & $28 / 40$ & 70.0 & $28 / 36$ & 77.8 & 73.7 \\
\hline Average corpus thickness & $25 / 39$ & 64.1 & $26 / 36$ & 72.2 & 68.0 \\
\hline Bimental breadth + average corpus thickness & $28 / 39$ & 71.8 & $30 / 36$ & 83.3 & 77.3 \\
\hline
\end{tabular}


$(68 \%)$. However, when both measurements are used in combination the overall accuracy rates reach $77.3 \%$.

The results show that males are more often misclassified than females: females achieved accuracy rates ranging from $72.2 \%$ $83.3 \%$ and males achieved accuracy rates ranging from $64.1 \%$ $71.8 \%$. This contradicts the statement by Weiss ${ }^{6}$ who reported that females were often misclassified as males within archaeological contexts due to the robusticity of the samples. It is possible that this has to do with the fact that the statement by Weiss was in reference to morphological methods. What was most surprising was that females produced accuracy rates around 10\% higher than males. This is seen in the case of Function 3, where the corpus thickness and average bimental breadth are being used in combination.

Identification of sex is a vital step in skeletal examinations. The flexibility to use different bones and fragmented bones is important, especially in forensic contexts. While there are many methods for the morphological assessment of skeletal remains, they are subjective and prone to high inter-observer error rates. Metric analysis is objective, enabling the reduction of inter- and intra-observer error. However, the difficulty in understanding the landmark and measurement definitions leads to metric analysis not being utilised as often as visual assessment. ${ }^{31}$

Most methods of sex determination using metrics on the mandible omit samples if there is tooth loss, bone resorption or pathologies on the basis that these conditions will affect the results. The sample used for this study contained some mandibles that had moderate levels of resorption, and in a few cases, abscesses; but none of these changes affect the location of the landmarks used in the method proposed. In addition, the promising accuracy rates achieved indicate that age and presence of dental pathologies play no role in the sexing of the mandible using these measurements. A further study on a larger sample would provide more solid evidence to support the proposed method.

\section{Conclusions}

While it was established that the method works and equations can be generated for the British Medieval population studied, improvements could be made through further research. For example, the study of the method on a different collection may help determine whether this technique is population-specific, or it can have a broader application. The addition of more measurements could potentially increase the overall accuracy rate of sex determination from the mandible; however this would probably be useful only if the mandible is complete. This study shows that bimental breadth and corpus thickness measurements are successful in the determination of sex in human skeletal remains: the measurements are quick and easy to obtain and produce promising results when used independently or in combination with each other.

\section{References}

1. Frayer DW, Wolpoff MH. Sexual dimorphism. Annu Rev Anthropol 1985;14:429-73.

2. Bass WM. Human osteology: a laboratory and field manual, 5th ed. Springfield, MO: Missouri Archaeological Society; 2005.

3. Meindl RM, Lovejoy CO, Mensforth RP, Carlos LD. Accuracy and direction of error in the sexing of the skeleton: implica- tions for paleodemographhy. Am J Phys Anthropol 1985;68:79-85.

4. Mays S, Cox M. Sex determination in skeletal remains. In: M Cox and S Mays (eds): Human osteology in archaeology and forensic science. London: Greenwich Medical Media; 2000. pp. 117-130.

5. Phenice TW. A newly developed visual method of sexing the os pubis. Am J Phys Anthropol 1969;30:297-301.

6. Weiss KM. On the systematic bias in skeletal sexing. Am J Phys Anthropol 1972;37:239-50.

7. Walker PL. Sexing skulls using discriminant function analysis of visually assessed traits. Am J Phys Anthropol 2008;136:39-50.

8. Steyn M, İşcan MY. Sexual dimorphism in the crania and mandibles of South African whites. Forens Sci Int 1998;98:9-16.

9. Hill CA. Technical note: evaluating mandibular ramus flexure as a morphological indicator of sex. Am J Phys Anthropol 2000;111:573-7.

10. Borrini M, Tomba Scalia GP, Mariani PP, et al. Taphonomy and anthropometry: the post mortem fate of measurement. A preliminary study on buried skulls - Proceedings of 19th IAFS World Meeting - Funchal Madeira, Portugal. Funchal-Madeira, Potugal, 12-17 September 2011.

11. Loth SR, Henneberg M. Mandibular ramus flexure: a new morphological indicator of sexual dimorphism in the human skeleton. Am J Phys Anthropol 1996;99:473-85.

12. Borrini M, Scalia Tomba GP. The postmortem fate of anthropometric measurements: taphonomic alteration of landmarks in buried skeletal remains. Proc Am Acad Forens Sci 2014;20:423-4.

13. Donnelly SM, Hens SM, Rogers NL, Schneider KL. Technical note: a blind test of mandibular ramus flexure as a morphologic indicator of sexual dimorphism in the human skeleton. Am J Phys Anthropol 1998;107:363-6.

14. Kemkes-Grottenthaler A, Löbin F, Stock F. Mandibular ramus flexure and gonial eversion as morphologic indicators of sex. J Compar Huma Biol 2002;53:97-111.

15. Thieme FP, Schull WJ. Sex determination from the skeleton. Human Biology 1957;29:242-73.

16. Reesink EM, Van Immerseel, AAH, Brand, R, Bruintjes TJD. Sexual dimorphism of the hyoid bone? Int J Osteoarchaeol 1999;9:357-60.

17. Purkait R. Measurement of ulna. A new method for determination of sex. J Forensic Sci 2001;46:924-7.

18. Koçak A, Aktas EÖ, Ertürk S, et al. Sex determination from the sternal end of the rib by osteometric analysis. Legal Med 2003;5:100-4.

19. Manolis SK, Eliopoulos C, Koilias CG, Fox SC. Sex determination using metacarpal biometric data from the Athens Collection. Forens Sci Int 2009;193:130-6.

20. Mountrakis C, Eliopoulos C, Koilias CG, Manolis SK. Sex determination using metatarsal osteometrics from the Athens collection. Forens Sci Int 2010;200:178.

21. Franklin D, O'Higgins P, Oxnard CE, Dadour I. Discriminant function sexing of the mandible of indigenous South Africans. Forens Sci Int 2008;179:84.

22. Kumar MP, Lokanadham S. Sex determination \& morphometric parameters of human mandible. Int J Res Med Sci 2013;1:93-6.

23. Burrell CL, Carpenter RJ. Analysis of human skeletal material from the poulton research project 1995-2012. The Poulton Project; 2013.

24. Atkin M, Garrod AP. Archaeology in Gloucester 1989. Transactions of the Bristol and Gloucestershire Archaeological Society 1990;108:185-92. 
25. Buikstra JE, Ubelaker DH. Standards for data collection from human skeletal remains. Research Series, no. 44. Fayetteville : Arkansas Archaeological Survey; 1994.

26. Martin R, Saller K. Lehrbuch der anthropologie. Stuttgart, Germany: Gustav Fischer Verlag; 1959.

27. Borrini M. Forensic anthropology: protocol and guidelines for the recovery and study of human remains. Phd thesis in "Evolutionary Biology and Ecology" XXIII cycle at University of Rome Tor Vergata; 2011.

28. Fairgrieve SI. Forensic cremation: recovery and analysis. Boca Raton, FL: CRC Press; 2008.
29. McKinley J. The analysis of cremated bone. In: M. Cox, S. Mays (Eds.), Human osteology in archaeology and forensic science. London: Greenwich Medical Media; 2000. pp. 403-421.

30. Symes SA, Rainwater CW, Chapman EN, et al. Patterned thermal destruction of human remains in a forensic setting-2. In: C.W. Schmidt and S.A. Symes (Eds.) The analysis of burned human remains. Amsterdam, the Netherlands: Academic Press; 2008. pp. 15-54.

31. Borrini M. Archeologia Forense: metodo e tecnica per il recupero dei resti umani: compendio per l'investigazione scientifica. Bologna: Lo Scarabeo; 2007. 